\title{
Synthesis, characterization and antitubercular evaluation of some new isoxazole appended 1-carboxamido-4,5- dihydro-1H-pyrazoles
}

\author{
Kishor PALLEAPATI 1 * (D) , Venkata Ramana KANCHARLAPALLI ${ }^{2}$ (D) , Afzal Basha SHAIK 3 (D) \\ K.J.R College of Pharmacy, Rajhamundry-533292, Andhra Pradesh, India. \\ ASN Pharmacy College, Tenali-522201, Andhra Pradesh, India. \\ Vignan Pharmacy College, Vadlamudi-522213, Andhra Pradesh, India. \\ Corresponding author. E-mail: kishorpharmcog@gmail.com (K.P.); Tel. +91-9966014374.
}

Received: 06 April 2018 / Revised: 09 August 2018 / Accepted: 12 September 2018

\begin{abstract}
Tuberculosis (TB) is an infectious disease caused by Mycobacterium tuberculosis. TB is a global problem and is one of the major culprits responsible for the death of many in the developing world despite the availability of more than 20 drugs. Hence, there is an imperative need for the development of novel antitubercular drugs. To develop better agents against tuberculosis, we synthesized a series of dihydropyrazole-1-carboxamides by the base-catalyzed condensation of isoxazolyl chalcones with semicarbazide. The compounds were purified by column chromatography and characterized by spectral methods including IR, ${ }^{1} \mathrm{H}$ NMR, ${ }^{13} \mathrm{C}$ NMR and elemental analysis. The antitubercular activity was screened against Mycobacterium tuberculosis by MABA assay where isoniazid was used as positive control for comparing the activity. The studies revealed that isoxazole-1-carboxamides were more active compared to the corresponding chalcones. Substituents on the phenyl ring at position- 5 of isoxazole ring played a crucial role in determining the antitubercular potency. The compounds $4 \mathbf{n}$ and $\mathbf{4 o}$ containing a blend of halogen and methoxyl groups at ortho and para positions of phenyl ring showed potency greater than the standard, isoniazid with $\mathrm{MIC}$ of $0.1 \mu \mathrm{g} / \mathrm{mL}$ and emerged as promising leads against TB. Further, $4 \mathrm{n}$ and $4 \mathrm{o}$ were assessed for their cytotoxic effects on L02 (human normal cell line) by using MTT assay. The compounds showed no cytotoxicity and established the safety of these compounds.
\end{abstract}

KEYWORDS: Isoxazolyl Chalcones; dihydropyrazole-1-carboxamides; antitubercular activity; Mycobacterium tuberculosis; MABA assay; MTT assay.

\section{INTRODUCTION}

Tuberculosis (TB) is one of the most important chronic communicable bacterial diseases caused by Mycobacterium tuberculosis. TB treatment is still an important problem in the developing world, despite the availability of a variety of antitubercular drugs. Global efforts to combat tuberculosis have saved an estimated 53 million lives since 2000 and reduced the TB mortality rate by 37\%, according to the Global TB Report 2017, released by the World Health Organization (WHO). Despite these achievements, the latest picture is depressing. TB remains the top infectious killer in 2016. TB is also the main cause of deaths related to antimicrobial resistance and the leading killer of people with HIV. Progress in most countries is stalling and is not fast enough to reach global targets or close persistent gaps in TB care and prevention [1]. This is due to the emergence of resistance and the toxicities associated with the existing chemotherapeutic agents. Hence, there is an urgent need for the development of novel molecules with less toxicity, short treatment protocols and also that can act through novel mechanism against the Mycobacterium tuberculosis bacterium.

Dihydropyrazole (Pyrazoline) is a heterocyclic scaffold of interest to medicinal chemists worldwide because of its ease of synthesis and a broad spectrum of bioactivities. In the recent past, scientists have reviewed [2-6] the pharmacological and physicochemical properties of dihydropyrazoles. Pyrazolines possess sundry of activities including antimicrobial [7-9], anticancer [10-12], antioxidant [13], antiamoebic [14], antiinflammatory [15], antidiabetic [16], diuretic [17] and carbonic anhydrase inhibition [18]. One of the extensively studied activities of dihydropyrazole-1-carboxamides is their antitubercular ability against different Mycobacterium species [19-24]. This may be due to the fact that the pyrazoline contains

How to cite this article: Pallepati K, Kancharlapalli VR, Shaik AB. Synthesis, characterization and antitubercular evaluation of some new isoxazole appended 1-carboxamido-4,5-dihydro-1H-pyrazoles. J Res Pharm. 2019; 23(2): 156-163. 
carbohydrazide portion which is also found in the most commonly used antitubercular drug, Isoniazid. The carbohydrazide portion of isoniazid is majorly responsible for the activity of Isoniazid. Isoxazole is an important heterocyclic ring present in its reduced form as isoxazolidine in the second line antitubercular drug, Cycloserine and its Schiff base analogue, Terizidone. Based on these observations, we predetermined to design some novel molecules that can show the duality of action similar to both isoniazid and cycloserine (Figure 1) with enhanced potency. In this paper we report the synthesis and antitubercular activity of a series of isoxazole appended 1-carboxamido-4,5-dihydro-1H-pyrazoles.<smiles>N[C@H]1CONC1=O</smiles>

Tautomers of Cycloserine<smiles>NNC(=O)c1ccncc1</smiles>

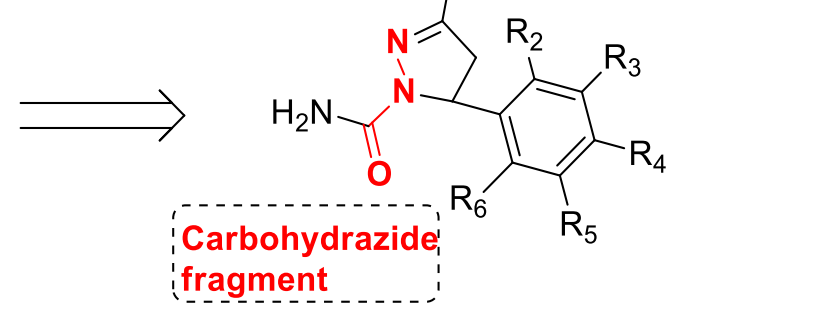

\section{Dihydropyrazole-1-carboxamides}

Isoniazid

Figure 1. Strategy employed in the design of isoxazole appended dihydropyrazole-1-carboxamides.

\section{RESULTS AND DISCUSSIONS}

\subsection{Chemistry}

The condensation of Isoxazolyl chalcones (3a-o) with semicarbazide with the aid of pyridine catalyst led to the formation of novel isoxazole linked 1-carboxamido-4,5-dihydro-1H-pyrazole derivatives (4a-o). Open tubular column chromatography helped to isolate the pure compounds. The IR and 1H NMR data untangled the structures of the purified compounds and the spectral data was in agreement with the predicted structures. The IR spectra showed three characteristic bands at the wave number regions $1619-1639 \mathrm{~cm}^{-1}$ $(\mathrm{C}=\mathrm{N}), 1662-1686(\mathrm{C}=\mathrm{O})$ and 3355-3399 $\left(-\mathrm{NH}_{2}\right)$ respectively. Three important peaks corresponding to $\mathrm{ABX}$ system of dihydropyrazole in the regions of $3.02-3.21 \mathrm{ppm}\left(1 \mathrm{H}, \mathrm{H}_{\mathrm{A}}, \mathrm{dd}, \mathrm{J}_{\mathrm{AX}}=3.6 \mathrm{~Hz}, \mathrm{dd}, \mathrm{J}_{\mathrm{AB}}=16 \mathrm{~Hz}\right), 3.75-3.90$ $\operatorname{ppm}\left(1 \mathrm{H}, \mathrm{H}_{\mathrm{B}}, \mathrm{dd}, \mathrm{J}_{\mathrm{AB}}=16 \mathrm{~Hz}, \mathrm{dd}, \mathrm{J}_{\mathrm{BX}}=12 \mathrm{~Hz}\right)$ and 5.19-5.44 $\mathrm{ppm}\left(1 \mathrm{H}, \mathrm{H}_{\mathrm{x}}, \mathrm{dd}, \mathrm{J}_{\mathrm{AX}}=3.6 \mathrm{~Hz}, \mathrm{dd}, \mathrm{J}_{\mathrm{BX}}=12 \mathrm{~Hz}\right)$ as well as a broad singlet at $\delta=10.20-10.51 \mathrm{ppm}$ for exchangeable $-\mathrm{NH}_{2}$ respectively in the $1 \mathrm{H}$ NMR spectrum established the structures of the 1-carboxamido-4,5-dihydro-1H-pyrazoles. The aromatic and methoxyl protons showed additional resonance signals. The molecular ion peak in positive ion mass spectrum further depicts the formation of dihydropyrazoles. Elemental analysis results were within $\pm 0.4 \%$ of the calculated values and helped in determining the elemental composition of the compounds.

\subsection{Antitubercular activity and SAR}

The preliminary in vitro antitubercular activity of the synthesized 1-carboxamido-4,5-dihydro- $1 \mathrm{H}$ pyrazoles (4a-4o) was performed by MABA assay using Isoniazid $(0.25 \mu \mathrm{g} / \mathrm{mL})$ as the positive control and the results are summarised in Table 1. Majority of the compounds were more potent than the preceding chalcones (Table 1) representing the positive contribution of dihydropyrazole carboxamide scaffold [25]. However, the activity of $4 \mathbf{c}, 4 \mathbf{d}$ and $4 \mathrm{~g}$ was similar to the chalcones $3 \mathrm{c}, 3 \mathrm{~d}$ and $3 \mathrm{~g}$ respectively. $4 \mathrm{n}$ and $4 \mathbf{o}$ were the most potent than Isoniazid with the MIC at $0.1 \mu \mathrm{g} / \mathrm{mL}$. This suggests that the compounds containing a combination of both halogens $(-\mathrm{Cl},-\mathrm{F})$ and methoxyl groups on the phenyl ring at position-5 of dihydropyrazole-1carboxamide plays a crucial role in enhancing the activity rather than the presence of just methoxyl groups. 
<smiles>[R]c1c([R])c([R])c(/C=C/C(=O)c2ccno2)c([R6])c1[R]</smiles>

(3a-o) (a)<smiles>[R]c1c([R5])c([R])c([C@H]2CC(c3ccno3)=NN2C(N)=O)c([R])c1[R]</smiles>

$(4 a-0)$

\begin{tabular}{|ll|}
\hline 3a: $\mathrm{R}_{2}=\mathrm{H} ; \mathrm{R}_{3}=\mathrm{H} ; \mathrm{R}_{4}=\mathrm{OCH}_{3} ; \mathrm{R}_{5}=\mathrm{H} ; \mathrm{R}_{6}=\mathrm{H}$ & 3h: $\mathrm{R}_{2}=\mathrm{H} ; \mathrm{R}_{3}=\mathrm{OCH}_{3} ; \mathrm{R}_{4}=\mathrm{OCH}_{3} ; \mathrm{R}_{5}=\mathrm{H} ; \mathrm{R}_{6}=\mathrm{H}$ \\
3b: $\mathrm{R}_{2}=\mathrm{H} ; \mathrm{R}_{3}=\mathrm{OCH}_{3} ; \mathrm{R}_{4}=\mathrm{H} ; \mathrm{R}_{5}=\mathrm{H} ; \mathrm{R}_{6}=\mathrm{H}$ & 3i: $\mathrm{R}_{2}=\mathrm{H} ; \mathrm{R}_{3}=\mathrm{OCH}_{3} ; \mathrm{R}_{4}=\mathrm{H} ; \mathrm{R}_{5}=\mathrm{OCH}_{3} ; \mathrm{R}_{6}=\mathrm{H}$ \\
3c: $\mathrm{R}_{2}=\mathrm{OCH}_{3} ; \mathrm{R}_{3}=\mathrm{H} ; \mathrm{R}_{4}=\mathrm{H} ; \mathrm{R}_{5}=\mathrm{H} ; \mathrm{R}_{6}=\mathrm{H}$ & 3j: $\mathrm{R}_{2}=\mathrm{OCH}_{3} ; \mathrm{R}_{3}=\mathrm{OCH}_{3} ; \mathrm{R}_{4}=\mathrm{OCH}_{3} ; \mathrm{R}_{5}=\mathrm{H} ; \mathrm{R}_{6}=\mathrm{H}$ \\
3d: $\mathrm{R}_{2}=\mathrm{OCH}_{3} ; \mathrm{R}_{3}=\mathrm{OCH}_{3} ; \mathrm{R}_{4}=\mathrm{H} ; \mathrm{R}_{5}=\mathrm{H} ; \mathrm{R}_{6}=\mathrm{H}$ & 3k: $\mathrm{R}_{2}=\mathrm{OCH}_{3} ; \mathrm{R}_{3}=\mathrm{H}_{2} \mathrm{R}_{4}=\mathrm{OCH}_{3} ; \mathrm{R}_{5}=\mathrm{OCH}_{3} ; \mathrm{R}_{6}=\mathrm{H}$ \\
3e: $\mathrm{R}_{2}=\mathrm{OCH}_{3} ; \mathrm{R}_{3}=\mathrm{H} ; \mathrm{R}_{4}=\mathrm{OCH}_{3} ; \mathrm{R}_{5}=\mathrm{H} ; \mathrm{R}_{6}=\mathrm{H}$ & 3I: $\mathrm{R}_{2}=\mathrm{OCH}_{3} ; \mathrm{R}_{3}=\mathrm{H} ; \mathrm{R}_{4}=\mathrm{OCH}_{3} ; \mathrm{R}_{5}=\mathrm{H} ; \mathrm{R}_{6}=\mathrm{OCH}_{3}$ \\
3f: $\mathrm{R}_{2}=\mathrm{OCH}_{3} ; \mathrm{R}_{3}=\mathrm{H} ; \mathrm{R}_{4}=\mathrm{H} ; \mathrm{R}_{5}=\mathrm{OCH}_{3} ; \mathrm{R}_{6}=\mathrm{H}$ & 3m: $\mathrm{R}_{2}=\mathrm{H} ; \mathrm{R}_{3}=\mathrm{OCH}_{3} ; \mathrm{R}_{4}=\mathrm{OCH}_{3} ; \mathrm{R}_{5}=\mathrm{OCH}_{3} ; \mathrm{R}_{6}=\mathrm{H}$ \\
3g: $\mathrm{R}_{2}=\mathrm{OCH}_{3} ; \mathrm{R}_{3}=\mathrm{H} ; \mathrm{R}_{4}=\mathrm{H} ; \mathrm{R}_{5}=\mathrm{H} ; \mathrm{R}_{6}=\mathrm{OCH}_{3}$ & 3n: $\mathrm{R}_{2}=\mathrm{F} ; \mathrm{R}_{3}=\mathrm{OCH}_{3} ; \mathrm{R}_{4}=\mathrm{OCH}_{3} ; \mathrm{R}_{5}=\mathrm{H} ; \mathrm{R}_{6}=\mathrm{H}$ \\
& 3o: $\mathrm{R}_{2}=\mathrm{Cl} ; \mathrm{R}_{3}=\mathrm{H} ; \mathrm{R}_{4}=\mathrm{OCH}_{3} ; \mathrm{R}_{5}=\mathrm{H} ; \mathrm{R}_{6}=\mathrm{OCH}_{3}$ \\
&
\end{tabular}

Figure 2. Synthesis of 1-carboxamido-4,5-dihydro-1H-pyrazole derivatives (4a-o). Reagents and conditions: Isoxazolyl chalcones (3a-o), (a) semicarbazide, ethanol, pyridine, reflux 6-8 h.

The potency of $4 \mathbf{n}$ and $4 \mathbf{o}$ was 20 and 10 folds compared to the chalcones $3 \mathbf{n}$ and 3o respectively. $4 \mathbf{l}$ $(\mathrm{MIC}=0.25 \mu \mathrm{g} / \mathrm{mL})$ showed similar potency to that of Isoniazid where as the activity of $4 \mathbf{e}$ was good with a MIC of $0.5 \mu \mathrm{g} / \mathrm{mL}$. Compounds $3 \mathbf{c}$ and $3 \mathbf{i}$ containing the methoxyl groups at the meta position on the phenyl ring exhibited poor action. The other compounds exhibited moderate activity with MIC ranging from 8 to 62.5 $\mu \mathrm{g} / \mathrm{mL}$. Structure-activity relationships indicated that the isoxazole ring clubbed to dihydropyrazole-1carboxamide is much important than the isoxazole connected to $a, \beta$-unsaturated carbonyl system for the activity. In addition, substitution of the ortho and para positions of the phenyl ring with a combination of halogen atoms and methoxyl groups is vital for the antitubercular activity than the presence of methoxyl substituents at meta position. The enhanced activity of is credited due to the presence of both $-\mathrm{N}-\mathrm{N}-\mathrm{C}=\mathrm{O}$ fragment and isoxazole ring found in Isoniazid and Cycloserine respectively. The proposed action may be due to the inhibition of both mycolic acid and peptidoglycan synthesis of the mycobacterial cell wall.

Table 1. Comparison of the results of the preliminary antitubercular activity of the dihydropyrazole-1carboxamides (4a-4o) with isoxazolyl chalcones (3a-3o).

\begin{tabular}{|c|c|c|c|}
\hline Compound & $\begin{array}{c}\text { MIC values }(\mu \mathrm{g} / \mathrm{mL}) \\
\text { vs. } \text { M. tuberculosis } \mathbf{H}_{37} \mathbf{R v}\end{array}$ & Compound & $\begin{array}{l}\text { MIC values }(\mu \mathrm{g} / \mathrm{mL}) \\
\text { vs. } \text { M. } \text { tuberculosis } \mathbf{H}_{37} \mathbf{R v}\end{array}$ \\
\hline $3 a$ & 62.5 & $4 a$ & 32 \\
\hline $3 b$ & 512 & $4 b$ & 62.5 \\
\hline $3 c$ & 126 & $4 c$ & 126 \\
\hline $3 d$ & 62.5 & $4 \mathrm{~d}$ & 62.5 \\
\hline $3 e$ & 16 & $4 \mathrm{e}$ & 0.5 \\
\hline $3 \mathrm{f}$ & 62.5 & $4 \mathrm{f}$ & 32 \\
\hline $3 g$ & 16 & $4 \mathrm{~g}$ & 16 \\
\hline $3 \mathrm{~h}$ & 126 & $4 \mathrm{~h}$ & 32 \\
\hline $3 \mathrm{i}$ & 252 & $4 \mathrm{i}$ & 126 \\
\hline $3 j$ & 16 & $4 j$ & 8 \\
\hline $3 k$ & 16 & $4 \mathrm{k}$ & 8 \\
\hline 31 & 2 & 41 & 0.25 \\
\hline $3 \mathrm{~m}$ & 32 & $4 \mathrm{~m}$ & 8 \\
\hline $3 n$ & 2 & $4 n$ & 0.1 \\
\hline 30 & 1 & 40 & 0.1 \\
\hline Isoniazid & 0.25 & & 0.25 \\
\hline
\end{tabular}




\subsection{Cytotoxicity studies}

The compounds $\mathbf{4 n}$ and $\mathbf{4 o}$ were evaluated for their in vitro cytotoxic activity against L02 (human normal cell line) by using MTT assay Mosmann's method. The result of the assay was displayed in Table 2. Both the compounds shown and $\mathrm{IC}_{50}$ value greater than $40 \mu \mathrm{g} / \mathrm{mL}$ representing that the compounds were non-toxic against the normal human cell lines.

Table 2. Cytotoxicity of compounds $4 \mathbf{n}$ and 40 against human normal cells $\left(\mathrm{IC}_{50} \pm \mathrm{SD}, \mu \mathrm{M}\right)$ a,b.

\begin{tabular}{ccc}
\hline S.No & Compounds & Human normal cells (L02) \\
\hline 1 & $4 \mathbf{n}$ & $>40$ \\
2 & $4 \mathbf{o}$ & $>40$ \\
\hline
\end{tabular}

a Mean value $\pm S D$ (standard deviation from three experiments).

b Boldface: $\mathrm{IC}_{50} \leq$ the control, $\left(\mathrm{IC}_{50}, \mu \mathrm{g} \mathrm{mL} \mathrm{m}^{-1}\right)$

\section{CONCLUSION}

In conclusion, we synthesized, purified and characterized a series of novel dihydropyrazole-1carboxamides. The compounds were evaluated for the antitubercular activity using MABA assay against Mycobacterium tuberculosis. Two compounds, $\mathbf{4 n}$ and $\mathbf{4 o}$ were more active than the standard Isoniazid and emerged as potential leads for the antitubercular activity. The activity results give an insight into the SAR features required for the antitubercular activity suggesting that the presence of both methoxyl and halogen substituents at positions-2,4,6 of phenyl ring present at the $5^{\text {th }}$ position are essential key for activity. No cytotoxicity was observed for the compounds $4 \mathbf{n}$ and $4 \mathbf{o}$ against the normal human cells. Further studies need to be carried out in order to determine the claimed in vivo antitubercular activity as well as the genotoxic studies to detect the toxicity of the most potent compounds on the DNA of human cells.

\section{MATERIALS AND METHODS}

\subsection{General}

Isoxazolyl chalcones previously synthesized [25] were used for the synthesis whereas semicarbazide was purchased from Sigma Aldrich Chemical Co. (Milwaukee, Wisconsin, USA). Merck grade silica gel-GF was used as the adsorbent for TLC to monitor the reactions. Boetius melting point apparatus was used to determine the melting points in open capillaries and the values are expressed in ${ }^{\circ} \mathrm{C}$ and are uncorrected. IR spectra were recorded on Bruker Vertex 80v spectrometer using potassium bromide disks and the wave numbers of the absorption bands are expressed in $\mathrm{cm}^{-1} .{ }^{1} \mathrm{H}$ NMR and ${ }^{13} \mathrm{C}$ NMR spectra were recorded on Bruker AMX $400 \mathrm{MHz}$ NMR spectrophotometer using TMS as an internal standard and the chemical shifts $(\delta)$ are expressed in ppm. MS spectra were recorded on Agilent LC-MS spectrometer and Carlo Erba 1108 elemental analyzer was used for the elemental analysis of $\mathrm{C}, \mathrm{H}$ and $\mathrm{N}$.

\subsection{Chemistry}

General method of synthesis of 1-carboxamido-4,5-dihydro-1H-pyrazole derivatives: The chalcones (3a-o) were prepared by reacting 1 -(isoxazole-5-yl)ethanone $(0.001$ mole) with appropriate aromatic aldehyde $(0.001$ mole) in ethanol $(7.5 \mathrm{~mL})$ and an aqueous solution of $\mathrm{KOH}(10 \%, 7.5 \mathrm{~mL})$ for $24 \mathrm{~h}$ at room temperature. The precipitate of the chalcone was then obtained by transferring the reaction mixture into crushed ice separately. The precipitate was filtered, washed thoroughly with water, dried and purified by recrystallized using either ethanol or chloroform as the solvents. 0.001 moles of (E)-1-(isoxazole-5-yl)-3-(substituted aryl)prop-2-en-1-one derivatives (3a-o) were dissolved in $7.5 \mathrm{ml}$ of ethanol. To this, catalytic amount of pyridine was added and then 0.0015 moles of semicarbazide was added and refluxed for about 6-8 h (Figure 2) [26-27]. After completion of the reaction, the mixture was transferred into the crushed ice to form a precipitate which was subjected to column chromatography to isolate the pure 1-carboxamido-4,5- dihydro- $1 \mathrm{H}$-pyrazole derivatives (4a-o).

3-(Isoxazol-5-yl)-5-(4-methoxyphenyl)-4,5-dihydro-1H-pyrazole-1-carboxamide (4a): Yield 56\%; Molecular Weight: 286.29; m.p. $166-168^{\circ} \mathrm{C}$; IR $\left(\mathrm{KBr}, \mathrm{cm}^{-1}\right)$ : $1622(\mathrm{C}=\mathrm{N}), 1677(\mathrm{C}=\mathrm{O}), 3367\left(-\mathrm{NH}_{2}\right)$; ${ }^{1} \mathrm{H}$ NMR $(400 \mathrm{MHz}$, $\left.\mathrm{CDCl}_{3}, \mathrm{ppm}\right): \delta 3.13\left(1 \mathrm{H}, \mathrm{H}_{\mathrm{A}}, \mathrm{dd}, \mathrm{J}_{\mathrm{AX}}=3.6 \mathrm{~Hz}, \mathrm{dd}, \mathrm{J}_{\mathrm{AB}}=16 \mathrm{~Hz}\right), 3.87\left(1 \mathrm{H}, \mathrm{H}_{\mathrm{B}}, \mathrm{dd}, \mathrm{J}_{\mathrm{AB}}=16 \mathrm{~Hz}, \mathrm{dd}, \mathrm{J}_{\mathrm{BX}}=12 \mathrm{~Hz}\right), 5.23$ $\left(1 \mathrm{H}, \mathrm{H}_{\mathrm{x}}, \mathrm{dd}, \mathrm{J}_{\mathrm{AX}}=3.6 \mathrm{~Hz}, \mathrm{dd}, \mathrm{J}_{\mathrm{BX}}=12 \mathrm{~Hz}\right), 10.44\left(2 \mathrm{H}, \mathrm{s}, \mathrm{NH}_{2}, \mathrm{D}_{2} \mathrm{O}\right.$ exchangeable $), 3.70\left(3 \mathrm{H}, \mathrm{s}, \mathrm{Ar}-\mathrm{OCH}_{3}\right), 6.15-8.59$ 
(6H, Ar-H); MS (m/z, \%): $287.1(\mathrm{M}+1,99.16)$; Anal. Calcd for: $\mathrm{C}_{14} \mathrm{H}_{14} \mathrm{~N}_{4} \mathrm{O}_{3}: \mathrm{C}, 58.73 ; \mathrm{H}, 4.93$; N, 19.57; Found: C, 59.18; H, 4.99; N, 20.16.

3-(Isoxazol-5-yl)-5-(3-methoxyphenyl)-4,5-dihydro-1H-pyrazole-1-carboxamide (4b): Yield 60\%; Molecular Weight: 286.29; m.p. 221-223 ${ }^{\circ} \mathrm{C}$; IR $\left(\mathrm{KBr}, \mathrm{cm}^{-1}\right)$ : $1634(\mathrm{C}=\mathrm{N}), 1686(\mathrm{C}=\mathrm{O}), 3391\left(-\mathrm{NH}_{2}\right)$; ${ }^{1} \mathbf{H}$ NMR $(400 \mathrm{MHz}$, $\left.\mathrm{CDCl}_{3}, \mathrm{ppm}\right): \delta 3.06\left(1 \mathrm{H}, \mathrm{H}_{\mathrm{A}}, \mathrm{dd}, \mathrm{J}_{\mathrm{AX}}=3.6 \mathrm{~Hz}, \mathrm{dd}, \mathrm{J}_{\mathrm{AB}}=16 \mathrm{~Hz}\right), 3.81\left(1 \mathrm{H}, \mathrm{H}_{\mathrm{B}}, \mathrm{dd}, \mathrm{J}_{\mathrm{AB}}=16 \mathrm{~Hz}, \mathrm{dd}, \mathrm{J}_{\mathrm{BX}}=12 \mathrm{~Hz}\right), 5.19$ $\left(1 \mathrm{H}, \mathrm{H}_{\mathrm{x}}, \mathrm{dd}, \mathrm{J}_{\mathrm{AX}}=3.6 \mathrm{~Hz}, \mathrm{dd}, \mathrm{J}_{\mathrm{BX}}=12 \mathrm{~Hz}\right), 10.25\left(2 \mathrm{H}, \mathrm{s}, \mathrm{NH}_{2}, \mathrm{D}_{2} \mathrm{O}\right.$ exchangeable), $3.85\left(3 \mathrm{H}, \mathrm{s}, \mathrm{Ar}-\mathrm{OCH}_{3}\right), 6.15-8.22$ $(6 \mathrm{H}, \mathrm{Ar}-\mathrm{H})$; MS (m/z, \%): $287.1(\mathrm{M}+1,99.22)$; Anal. Calcd for: $\mathrm{C}_{14} \mathrm{H}_{14} \mathrm{~N}_{4} \mathrm{O}_{3}$ : C, 58.73; H, 4.93; N, 19.57; Found: C, 59.18; H, 4.99; N, 20.16.

3-(Isoxazol-5-yl)-5-(2-methoxyphenyl)-4,5-dihydro-1H-pyrazole-1-carboxamide (4c): Yield 58\%; Molecular Weight: 286.29; m.p. 256-258 ${ }^{\circ} \mathrm{C}$; IR (KBr, cm $\left.{ }^{-1}\right)$ : $1619(\mathrm{C}=\mathrm{N}), 1667(\mathrm{C}=\mathrm{O}), 3375\left(-\mathrm{NH}_{2}\right) ;{ }^{1} \mathbf{H} \mathbf{N M R}(400 \mathrm{MHz}$, $\left.\mathrm{CDCl}_{3}, \mathrm{ppm}\right): \delta 3.14\left(1 \mathrm{H}, \mathrm{H}_{\mathrm{A}}, \mathrm{dd}, \mathrm{J}_{\mathrm{AX}}=3.6 \mathrm{~Hz}, \mathrm{dd}, \mathrm{J}_{\mathrm{AB}}=16 \mathrm{~Hz}\right), 3.82\left(1 \mathrm{H}, \mathrm{H}_{\mathrm{B}}, \mathrm{dd}, \mathrm{J}_{\mathrm{AB}}=16 \mathrm{~Hz}, \mathrm{dd}, \mathrm{J}_{\mathrm{BX}}=12 \mathrm{~Hz}\right), 5.25$ $\left(1 \mathrm{H}, \mathrm{H}_{\mathrm{x}}, \mathrm{dd}, \mathrm{J}_{\mathrm{AX}}=3.6 \mathrm{~Hz}, \mathrm{dd}, \mathrm{J}_{\mathrm{BX}}=12 \mathrm{~Hz}\right), 10.41\left(2 \mathrm{H}, \mathrm{s}, \mathrm{NH}_{2}, \mathrm{D}_{2} \mathrm{O}\right.$ exchangeable), $3.72(3 \mathrm{H}, \mathrm{s}, \mathrm{Ar}-\mathrm{OCH})_{3}, 6.23-8.05$ $(6 \mathrm{H}, \mathrm{Ar}-\mathrm{H})$; MS (m/z, \%): 287.1 (M+1, 99.09); Anal. Calcd for: $\mathrm{C}_{14} \mathrm{H}_{14} \mathrm{~N}_{4} \mathrm{O}_{3}$ : C, 58.73; H, 4.93; N, 19.57; Found: C, 59.18; H, 4.99; N, 20.16.

3-(Isoxazol-5-yl)-5-(2,3-dimethoxyphenyl)-4,5-dihydro-1H-pyrazole-1-carboxamide (4d): Yield 52\%; Molecular Weight: 316.31; m.p. 210-212 ${ }^{\circ} \mathrm{C}$; IR (KBr, cm $\left.{ }^{-1}\right)$ : 1629 (C=N), 1669 (C=O), 3359 (-NH $)$; ${ }^{1} \mathbf{H ~ N M R}$ (400 $\left.\mathrm{MHz}_{\mathrm{CDCl}}, \mathrm{ppm}\right): \delta 3.18\left(1 \mathrm{H}, \mathrm{H}_{\mathrm{A}}, \mathrm{dd}, \mathrm{J}_{\mathrm{AX}}=3.6 \mathrm{~Hz}, \mathrm{dd}, \mathrm{J}_{\mathrm{AB}}=16 \mathrm{~Hz}\right), 3.85\left(1 \mathrm{H}, \mathrm{H}_{\mathrm{B}}, \mathrm{dd}, \mathrm{J}_{\mathrm{AB}}=16 \mathrm{~Hz}, \mathrm{dd}, \mathrm{J}_{\mathrm{BX}}=12 \mathrm{~Hz}\right)$, $5.19\left(1 \mathrm{H}, \mathrm{H}_{\mathrm{x}}, \mathrm{dd}, \mathrm{J}_{\mathrm{Ax}}=3.6 \mathrm{~Hz}, \mathrm{dd}, \mathrm{J}_{\mathrm{BX}}=12 \mathrm{~Hz}\right), 10.49\left(2 \mathrm{H}, \mathrm{s}, \mathrm{NH}_{2}, \mathrm{D}_{2} \mathrm{O}\right.$ exchangeable $), 3.53(3 \mathrm{H}, \mathrm{s}, \mathrm{Ar}-\mathrm{OCH} 3), 3.68$ $\left(3 \mathrm{H}, \mathrm{s}, \mathrm{Ar}-\mathrm{OCH}_{3}\right), 6.35-8.28(5 \mathrm{H}, \mathrm{Ar}-\mathrm{H})$; MS (m/z, \%): 317.1 (M+1, 99.06); Anal. Calcd for: $\mathrm{C}_{15} \mathrm{H}_{16} \mathrm{~N}_{4} \mathrm{O}_{4}: \mathrm{C}_{,} 56.69$; H, 5.10; N, 17.71; Found: C, 56.88; H, 5.31; N, 17.99 .

3-(Isoxazol-5-yl)-5-(2,4-dimethoxyphenyl)-4,5-dihydro-1H-pyrazole-1-carboxamide (4e): Yield 65\%; Molecular Weight: 316.31; m.p. 189-191 ${ }^{\circ} \mathrm{C}$; IR (KBr, cm $\left.{ }^{-1}\right)$ : 1631 (C=N), 1682 (C=O), $3361\left(-\mathrm{NH}_{2}\right)$; ${ }^{1} \mathbf{H ~ N M R}$ (400 $\left.\mathrm{MHz}_{\mathrm{CDCl}}, \mathrm{CDpm}_{3}\right): \delta 3.10\left(1 \mathrm{H}, \mathrm{H}_{\mathrm{A}}, \mathrm{dd}, \mathrm{J}_{\mathrm{AX}}=3.6 \mathrm{~Hz}, \mathrm{dd}, \mathrm{J}_{\mathrm{AB}}=16 \mathrm{~Hz}\right), 3.90\left(1 \mathrm{H}, \mathrm{H}_{\mathrm{B}}, \mathrm{dd}, \mathrm{J}_{\mathrm{AB}}=16 \mathrm{~Hz}, \mathrm{dd}, \mathrm{J}_{\mathrm{BX}}=12 \mathrm{~Hz}\right)$, $5.22\left(1 \mathrm{H}, \mathrm{H}_{\mathrm{x}}, \mathrm{dd}, \mathrm{J}_{\mathrm{AX}}=3.6 \mathrm{~Hz}, \mathrm{dd}, \mathrm{J}_{\mathrm{Bx}}=12 \mathrm{~Hz}\right), 10.35\left(2 \mathrm{H}, \mathrm{s}, \mathrm{NH}_{2}, \mathrm{D}_{2} \mathrm{O}\right.$ exchangeable $), 3.51\left(3 \mathrm{H}, \mathrm{s}, \mathrm{Ar}-\mathrm{OCH}_{3}\right), 3.72$ $\left(3 \mathrm{H}, \mathrm{s}, \mathrm{Ar}-\mathrm{OCH}_{3}\right), 6.18-8.10(5 \mathrm{H}, \mathrm{Ar}-\mathrm{H})$; MS (m/z, \%): 317.1 (M+1, 99.11); Anal. Calcd for: $\mathrm{C}_{15} \mathrm{H}_{16} \mathrm{~N}_{4} \mathrm{O}_{4}: \mathrm{C}, 56.69$; H, 5.10; N, 17.71; Found: C, 56.88; H, 5.31; N, 17.99 .

3-(Isoxazol-5-yl)-5-(2,5-dimethoxyphenyl)-4,5-dihydro-1H-pyrazole-1-carboxamide (4f): Yield 59\%;

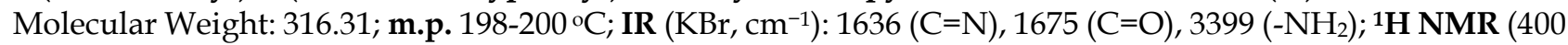
$\left.\mathrm{MHz}, \mathrm{CDCl}_{3}, \mathrm{ppm}\right): \delta 3.15\left(1 \mathrm{H}, \mathrm{H}_{\mathrm{A}}, \mathrm{dd}, \mathrm{J}_{\mathrm{AX}}=3.6 \mathrm{~Hz}, \mathrm{dd}, \mathrm{J}_{\mathrm{AB}}=16 \mathrm{~Hz}\right), 3.85\left(1 \mathrm{H}, \mathrm{H}_{\mathrm{B}}, \mathrm{dd}, \mathrm{J}_{\mathrm{AB}}=16 \mathrm{~Hz}, \mathrm{dd}, \mathrm{J}_{\mathrm{BX}}=12 \mathrm{~Hz}\right)$, $5.21\left(1 \mathrm{H}, \mathrm{H}_{\mathrm{x}}, \mathrm{dd}, \mathrm{J}_{\mathrm{AX}}=3.6 \mathrm{~Hz}, \mathrm{dd}, \mathrm{J}_{\mathrm{BX}}=12 \mathrm{~Hz}\right), 10.18\left(2 \mathrm{H}, \mathrm{s}, \mathrm{NH}_{2}, \mathrm{D}_{2} \mathrm{O}\right.$ exchangeable $), 3.66(3 \mathrm{H}, \mathrm{s}, \mathrm{Ar}-\mathrm{OCH} 3), 3.74$ $\left(3 \mathrm{H}, \mathrm{s}, \mathrm{Ar}-\mathrm{OCH}_{3}\right), 6.09-8.18(5 \mathrm{H}, \mathrm{Ar}-\mathrm{H})$; MS (m/z, \%): $317.1(\mathrm{M}+1,99.16)$; Anal. Calcd for: $\mathrm{C}_{15} \mathrm{H}_{16} \mathrm{~N}_{4} \mathrm{O}_{4}: \mathrm{C}_{\text {, } 56.69}$; H, 5.10; N, 17.71; Found: C, 56.88; H, 5.31; N, 17.99 .

3-(Isoxazol-5-yl)-5-(2,6-dimethoxyphenyl)-4,5-dihydro-1H-pyrazole-1-carboxamide (4g): Yield 45\%; Molecular Weight: 316.31; m.p. 282-284 ${ }^{\circ} \mathrm{C}$; IR (KBr, cm $\left.{ }^{-1}\right)$ : 1626 (C=N), $1671(\mathrm{C}=\mathrm{O}), 3361\left(-\mathrm{NH}_{2}\right)$; ${ }^{1} \mathrm{H}_{\mathrm{NMR}}(400$ $\left.\mathrm{MHz}_{\mathrm{CDCl}}, \mathrm{CDpm}_{3}\right): \delta 3.13\left(1 \mathrm{H}, \mathrm{H}_{\mathrm{A}}, \mathrm{dd}, \mathrm{J}_{\mathrm{AX}}=3.6 \mathrm{~Hz}, \mathrm{dd}, \mathrm{J}_{\mathrm{AB}}=16 \mathrm{~Hz}\right), 3.86\left(1 \mathrm{H}, \mathrm{H}_{\mathrm{B}}, \mathrm{dd}, \mathrm{J}_{\mathrm{AB}}=16 \mathrm{~Hz}, \mathrm{dd}, \mathrm{J}_{\mathrm{BX}}=12 \mathrm{~Hz}\right)$, $5.29\left(1 \mathrm{H}, \mathrm{H}_{\mathrm{x}}, \mathrm{dd}, \mathrm{J}_{\mathrm{AX}}=3.6 \mathrm{~Hz}, \mathrm{dd}, \mathrm{J}_{\mathrm{BX}}=12 \mathrm{~Hz}\right), 10.46\left(2 \mathrm{H}, \mathrm{s}, \mathrm{NH}_{2}, \mathrm{D}_{2} \mathrm{O}\right.$ exchangeable $), 3.58(3 \mathrm{H}, \mathrm{s}, \mathrm{Ar}-\mathrm{OCH} 3), 3.78$ $\left(3 \mathrm{H}, \mathrm{s}, \mathrm{Ar}-\mathrm{OCH}_{3}\right), 6.07-8.23(5 \mathrm{H}, \mathrm{Ar}-\mathrm{H})$; MS (m/z, \%): 317.1 (M+1, 99.01); Anal. Calcd for: $\mathrm{C}_{15} \mathrm{H}_{16} \mathrm{~N}_{4} \mathrm{O}_{4}: \mathrm{C}_{\text {, } 56.69}$; H, 5.10; N, 17.71; Found: C, 56.88; H, 5.31; N, 17.99 .

3-(Isoxazol-5-yl)-5-(3,4-dimethoxyphenyl)-4,5-dihydro-1H-pyrazole-1-carboxamide (4h): Yield 55\%; Molecular Weight: 316.31; m.p. 246-248 ${ }^{\circ} \mathrm{C}$; IR (KBr, cm $\left.{ }^{-1}\right)$ : $1624(\mathrm{C}=\mathrm{N}), 1678(\mathrm{C}=\mathrm{O}), 3356\left(-\mathrm{NH}_{2}\right)$; $^{\mathbf{1}} \mathrm{H}_{\mathrm{NMR}}$ (400 $\left.\mathrm{MHz}_{\mathrm{CDCl}}, \mathrm{Cpm}\right): \delta 3.20\left(1 \mathrm{H}, \mathrm{H}_{\mathrm{A}}, \mathrm{dd}, \mathrm{J}_{\mathrm{AX}}=3.6 \mathrm{~Hz}, \mathrm{dd}, \mathrm{J}_{\mathrm{AB}}=16 \mathrm{~Hz}\right), 3.79\left(1 \mathrm{H}, \mathrm{H}_{\mathrm{B}}, \mathrm{dd}, \mathrm{J}_{\mathrm{AB}}=16 \mathrm{~Hz}, \mathrm{dd}, \mathrm{J}_{\mathrm{BX}}=12 \mathrm{~Hz}\right)$, $5.30\left(1 \mathrm{H}, \mathrm{H}_{\mathrm{x}}, \mathrm{dd}, \mathrm{J}_{\mathrm{AX}}=3.6 \mathrm{~Hz}, \mathrm{dd}, \mathrm{J}_{\mathrm{BX}}=12 \mathrm{~Hz}\right), 10.51\left(2 \mathrm{H}, \mathrm{s}, \mathrm{NH}_{2}, \mathrm{D}_{2} \mathrm{O}\right.$ exchangeable $), 3.61\left(3 \mathrm{H}, \mathrm{s}, \mathrm{Ar}-\mathrm{OCH}_{3}\right), 3.83$ $\left(3 \mathrm{H}, \mathrm{s}, \mathrm{Ar}-\mathrm{OCH}_{3}\right), 6.25-8.46(5 \mathrm{H}, \mathrm{Ar}-\mathrm{H})$; $\mathrm{MS}(\mathrm{m} / \mathrm{z}, \%): 317.1(\mathrm{M}+1,99.26)$; Anal. Calcd for: $\mathrm{C}_{15} \mathrm{H}_{16} \mathrm{~N}_{4} \mathrm{O}_{4}: \mathrm{C}_{,} 56.69$; H, 5.10; N, 17.71; Found: C, 56.88; H, 5.31; N, 17.99 .

3-(Isoxazol-5-yl)-5-(3,5-dimethoxyphenyl)-4,5-dihydro-1H-pyrazole-1-carboxamide (4i): Yield 48\%;

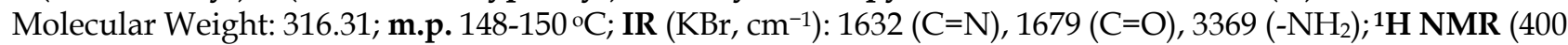
$\left.\mathrm{MHz}_{\mathrm{CDCl}}, \mathrm{ppm}\right): \delta 3.21\left(1 \mathrm{H}, \mathrm{H}_{\mathrm{A}}, \mathrm{dd}, \mathrm{J}_{\mathrm{AX}}=3.6 \mathrm{~Hz}, \mathrm{dd}, \mathrm{J}_{\mathrm{AB}}=16 \mathrm{~Hz}\right), 3.81\left(1 \mathrm{H}, \mathrm{H}_{\mathrm{B}}, \mathrm{dd}, \mathrm{J}_{\mathrm{AB}}=16 \mathrm{~Hz}, \mathrm{dd}, \mathrm{J}_{\mathrm{BX}}=12 \mathrm{~Hz}\right)$, $5.33\left(1 \mathrm{H}, \mathrm{H}_{\mathrm{x}}, \mathrm{dd}, \mathrm{J}_{\mathrm{AX}}=3.6 \mathrm{~Hz}, \mathrm{dd}, \mathrm{J}_{\mathrm{BX}}=12 \mathrm{~Hz}\right), 10.48\left(2 \mathrm{H}, \mathrm{s}, \mathrm{NH}_{2}, \mathrm{D}_{2} \mathrm{O}\right.$ exchangeable $), 3.51(3 \mathrm{H}, \mathrm{s}, \mathrm{Ar}-\mathrm{OCH} 3), 3.99$ 
$\left(3 \mathrm{H}, \mathrm{s}, \mathrm{Ar}-\mathrm{OCH}_{3}\right), 6.33-8.61(5 \mathrm{H}, \mathrm{Ar}-\mathrm{H})$; $\mathrm{MS}(\mathrm{m} / z, \%): 317.1(\mathrm{M}+1,99.08)$; Anal. Calcd for: $\mathrm{C}_{15} \mathrm{H}_{16} \mathrm{~N}_{4} \mathrm{O}_{4}: \mathrm{C}, 56.69$; $\mathrm{H}, 5.10$; N, 17.71; Found: C, 56.88; H, 5.31; N, 17.99 .

3-(Isoxazol-5-yl)-5-(2,3,4-trimethoxyphenyl)-4,5-dihydro-1H-pyrazole-1-carboxamide (4j): Yield $60 \%$; Molecular Weight: 346.13; m.p. 262-264 ${ }^{\circ} \mathrm{C}$; IR $1639(\mathrm{C}=\mathrm{N}), 1662(\mathrm{C}=\mathrm{O}), 3355\left(-\mathrm{NH}_{2}\right)$; ${ }^{1} \mathrm{H}$ NMR $(400 \mathrm{MHz}$, $\left.\mathrm{CDCl}_{3}, \mathrm{ppm}\right): \delta 3.09\left(1 \mathrm{H}, \mathrm{H}_{\mathrm{A}}, \mathrm{dd}, \mathrm{J}_{\mathrm{AX}}=3.6 \mathrm{~Hz}, \mathrm{dd}, \mathrm{J}_{\mathrm{AB}}=16 \mathrm{~Hz}\right), 3.82\left(1 \mathrm{H}, \mathrm{H}_{\mathrm{B}}, \mathrm{dd}, \mathrm{J}_{\mathrm{AB}}=16 \mathrm{~Hz}, \mathrm{dd}, \mathrm{J}_{\mathrm{BX}}=12 \mathrm{~Hz}\right), 5.40$ $\left(1 \mathrm{H}, \mathrm{H}_{\mathrm{x}}, \mathrm{dd}, \mathrm{J}_{\mathrm{AX}}=3.6 \mathrm{~Hz}, \mathrm{dd}, \mathrm{J}_{\mathrm{BX}}=12 \mathrm{~Hz}\right), 10.39\left(2 \mathrm{H}, \mathrm{s}, \mathrm{NH}_{2}, \mathrm{D}_{2} \mathrm{O}\right.$ exchangeable $), 3.65\left(3 \mathrm{H}, \mathrm{s}, \mathrm{Ar}-\mathrm{OCH}_{3}\right), 3.99(6 \mathrm{H}$, $\left.\mathrm{s}, 2 \mathrm{x} \mathrm{Ar}-\mathrm{OCH}_{3}\right), 6.36-8.44(4 \mathrm{H}, \mathrm{Ar}-\mathrm{H})$; $\mathrm{MS}(\mathrm{m} / \mathrm{z}, \%)$ : $346.1(\mathrm{M}+1,99.14)$; Anal. Calcd for: $\mathrm{C}_{16} \mathrm{H}_{18} \mathrm{~N}_{4} \mathrm{O}_{5}$ : C, 55.49; H, 5.24; N, 16.18; Found: C, 55.89; H, 5.65; N, 16.52 .

3-(Isoxazol-5-yl)-5-(2,4,5-trimethoxyphenyl)-4,5-dihydro-1H-pyrazole-1-carboxamide (4k): Yield 51\%; Molecular Weight: 346.13; m.p. 231-233 ${ }^{\circ} \mathrm{C}$; IR $\left(\mathrm{KBr}, \mathrm{cm}^{-1}\right)$ : $1625(\mathrm{C}=\mathrm{N}), 1672(\mathrm{C}=\mathrm{O}), 3362\left(-\mathrm{NH}_{2}\right) ;{ }^{1} \mathbf{H}$ NMR $(400$ $\left.\mathrm{MHz}, \mathrm{CDCl}_{3}, \mathrm{ppm}\right): \delta 3.09\left(1 \mathrm{H}, \mathrm{H}_{\mathrm{A}}, \mathrm{dd}, \mathrm{J}_{\mathrm{AX}}=3.6 \mathrm{~Hz}, \mathrm{dd}, \mathrm{J}_{\mathrm{AB}}=16 \mathrm{~Hz}\right), 3.75\left(1 \mathrm{H}, \mathrm{H}_{\mathrm{B}}, \mathrm{dd}, \mathrm{J}_{\mathrm{AB}}=16 \mathrm{~Hz}, \mathrm{dd}, \mathrm{J}_{\mathrm{BX}}=12 \mathrm{~Hz}\right)$, $5.44\left(1 \mathrm{H}, \mathrm{H}_{\mathrm{x}}, \mathrm{dd}, \mathrm{J}_{\mathrm{AX}}=3.6 \mathrm{~Hz}, \mathrm{dd}, \mathrm{J}_{\mathrm{BX}}=12 \mathrm{~Hz}\right), 10.40\left(2 \mathrm{H}, \mathrm{s}, \mathrm{NH}_{2}, \mathrm{D}_{2} \mathrm{O}\right.$ exchangeable $), 3.80\left(3 \mathrm{H}, \mathrm{s}, \mathrm{Ar}-\mathrm{OCH}_{3}\right), 3.96$ $\left(6 \mathrm{H}, \mathrm{s}, 2 \mathrm{Ar}-\mathrm{OCH}_{3}\right), 6.56-8.82(4 \mathrm{H}, \mathrm{Ar}-\mathrm{H})$; Anal. Calcd for: $\mathrm{C}_{16} \mathrm{H}_{18} \mathrm{~N}_{4} \mathrm{O}_{5}: \mathrm{C}, 55.49 ; \mathrm{H}, 5.24 ; \mathrm{N}, 16.18$; Found: $\mathrm{C}$, $55.89 ; \mathrm{H}, 5.65 ; \mathrm{N}, 16.52$.

3-(Isoxazol-5-yl)-5-(2,4,6-trimethoxyphenyl)-4,5-dihydro-1H-pyrazole-1-carboxamide (41): Yield 41\%; Molecular Weight: 346.13; m.p. 272-274 ${ }^{\circ} \mathrm{C}$; IR $\left(\mathrm{KBr}, \mathrm{cm}^{-1}\right)$ : $1635(\mathrm{C}=\mathrm{N}), 1681(\mathrm{C}=\mathrm{O}), 3382\left(-\mathrm{NH}_{2}\right) ;{ }^{1} \mathbf{H}$ NMR $(400$ $\left.\mathrm{MHz}, \mathrm{CDCl}_{3}, \mathrm{ppm}\right): \delta 3.13\left(1 \mathrm{H}, \mathrm{H}_{\mathrm{A}}, \mathrm{dd}, \mathrm{J}_{\mathrm{AX}}=3.6 \mathrm{~Hz}, \mathrm{dd}, \mathrm{J}_{\mathrm{AB}}=16 \mathrm{~Hz}\right), 3.85\left(1 \mathrm{H}, \mathrm{H}_{\mathrm{B}}, \mathrm{dd}, \mathrm{J}_{\mathrm{AB}}=16 \mathrm{~Hz}, \mathrm{dd}, \mathrm{J}_{\mathrm{BX}}=12 \mathrm{~Hz}\right)$, $5.20\left(1 \mathrm{H}, \mathrm{H}_{\mathrm{x}}, \mathrm{dd}, \mathrm{J}_{\mathrm{AX}}=3.6 \mathrm{~Hz}, \mathrm{dd}, \mathrm{J}_{\mathrm{BX}}=12 \mathrm{~Hz}\right), 10.32\left(2 \mathrm{H}, \mathrm{s}, \mathrm{NH}_{2}, \mathrm{D}_{2} \mathrm{O}\right.$ exchangeable $), 3.79\left(3 \mathrm{H}, \mathrm{s}, \mathrm{Ar}-\mathrm{OCH}_{3}\right), 3.91$ $\left(6 \mathrm{H}, \mathrm{s}, 2 \mathrm{Ar}-\mathrm{OCH}_{3}\right), 6.35-8.60(4 \mathrm{H}, \mathrm{Ar}-\mathrm{H})$; $\mathrm{MS}(\mathrm{m} / \mathrm{z}, \%): 346.1(\mathrm{M}+1,99.09)$; Anal. Calcd for: $\mathrm{C}_{16} \mathrm{H}_{18} \mathrm{~N}_{4} \mathrm{O}_{5}$ : C, 55.49; H, 5.24; N, 16.18; Found: C, 55.89; H, 5.65; N, 16.52.

3-(Isoxazol-5-yl)-5-(3,4,5-trimethoxyphenyl)-4,5-dihydro-1H-pyrazole-1-carboxamide (4m): Yield 71\%; Molecular Weight: 346.13; m.p. 291-293 ${ }^{\circ} \mathrm{C}$; IR $\left(\mathrm{KBr}, \mathrm{cm}^{-1}\right)$ : $1628(\mathrm{C}=\mathrm{N}), 1685(\mathrm{C}=\mathrm{O}), 3369\left(-\mathrm{NH}_{2}\right) ;{ }^{\mathbf{1}} \mathrm{H}$ NMR $(400$ $\left.\mathrm{MHz}, \mathrm{CDCl}_{3}, \mathrm{ppm}\right): \delta 3.11\left(1 \mathrm{H}, \mathrm{H}_{\mathrm{A}}, \mathrm{dd}, \mathrm{J}_{\mathrm{AX}}=3.6 \mathrm{~Hz}, \mathrm{dd}, \mathrm{J}_{\mathrm{AB}}=16 \mathrm{~Hz}\right), 3.80\left(1 \mathrm{H}, \mathrm{H}_{\mathrm{B}}, \mathrm{dd}, \mathrm{J}_{\mathrm{AB}}=16 \mathrm{~Hz}, \mathrm{dd}, \mathrm{J}_{\mathrm{BX}}=12 \mathrm{~Hz}\right)$, $5.15\left(1 \mathrm{H}, \mathrm{H}_{\mathrm{x}}, \mathrm{dd}, \mathrm{J}_{\mathrm{AX}}=3.6 \mathrm{~Hz}, \mathrm{dd}, \mathrm{J}_{\mathrm{BX}}=12 \mathrm{~Hz}\right), 10.20\left(2 \mathrm{H}, \mathrm{s}, \mathrm{NH}_{2}, \mathrm{D}_{2} \mathrm{O}\right.$ exchangeable), $3.70\left(3 \mathrm{H}, \mathrm{s}, \mathrm{Ar}_{-} \mathrm{OCH}_{3}\right), 3.95$ $\left(6 \mathrm{H}, \mathrm{s}, 2 \mathrm{x} \mathrm{Ar}-\mathrm{OCH}_{3}\right), 6.11-8.33(4 \mathrm{H}, \mathrm{Ar}-\mathrm{H})$; MS $(\mathrm{m} / \mathrm{z}, \%)$ : $346.1(\mathrm{M}+1,99.20)$; Anal. Calcd for: $\mathrm{C}_{15} \mathrm{H}_{15} \mathrm{NO}_{5}$ : C, 62.28; H, 5.23; N, 4.84; Found: C, 62.55; H, 5.44; N, 5.85 .

3-(Isoxazol-5-yl)-5-(2-fluoro-3,4-dimethoxyphenyl)-4,5-dihydro-1H-pyrazole-1-carboxamide (4n): Yield 81\%; Molecular Weight: 334.11; m.p. 302-304 ${ }^{\circ} \mathrm{C}$; IR (KBr, cm $\left.{ }^{-1}\right): 1630(\mathrm{C}=\mathrm{N}), 1662(\mathrm{C}=\mathrm{O}), 3360\left(-\mathrm{NH}_{2}\right)$; ${ }^{1} \mathbf{H}$ NMR $\left(400 \mathrm{MHz}, \mathrm{CDCl}_{3}, \mathrm{ppm}\right): \delta 3.06\left(1 \mathrm{H}, \mathrm{H}_{\mathrm{A}}, \mathrm{dd}, \mathrm{J}_{\mathrm{AX}}=3.6 \mathrm{~Hz}, \mathrm{dd}, \mathrm{J}_{\mathrm{AB}}=16 \mathrm{~Hz}\right), 3.81\left(1 \mathrm{H}, \mathrm{H}_{\mathrm{B}}, \mathrm{dd}, \mathrm{J}_{\mathrm{AB}}=16 \mathrm{~Hz}, \mathrm{dd}, \mathrm{J}_{\mathrm{BX}}=\right.$ $12 \mathrm{~Hz}), 5.31\left(1 \mathrm{H}, \mathrm{H}_{\mathrm{x}}, \mathrm{dd}, \mathrm{J}_{\mathrm{AX}}=3.6 \mathrm{~Hz}, \mathrm{dd}, \mathrm{J}_{\mathrm{BX}}=12 \mathrm{~Hz}\right), 10.14\left(2 \mathrm{H}, \mathrm{s}, \mathrm{NH}_{2}, \mathrm{D}_{2} \mathrm{O}\right.$ exchangeable), $3.61(3 \mathrm{H}, \mathrm{s}, \mathrm{Ar}-$ $\left.\mathrm{OCH}_{3}\right), 3.89\left(3 \mathrm{H}, \mathrm{s}, 2 \mathrm{x} \mathrm{Ar}-\mathrm{OCH}_{3}\right), 6.22-8.41(4 \mathrm{H}, \mathrm{Ar}-\mathrm{H}) ;{ }^{13} \mathrm{C}$ NMR $(100 \mathrm{MHz}, \mathrm{CDCl} 3, \mathrm{ppm}): \delta 155.62$ (C-3), 39.54 (C-4), 62.50 (C-5), $155.12\left(\mathrm{CONH}_{2}\right), 150.06$ (C-3 of Isoxazolyl ring), 100.52 (C-4 of Isoxazolyl ring), 155.66 (C-5 of Isoxazolyl ring), 102.11 (C-1 of phenyl ring), 145.12 (C-2 of phenyl ring), 134.24 (C-3 of phenyl ring), 149.42

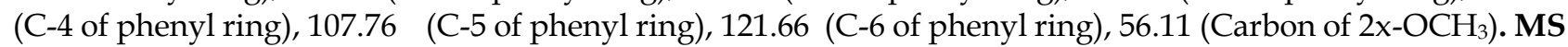
$(\mathrm{m} / \mathrm{z}, \%): 335.1$ (M+1, 99.03); Anal. Calcd for: $\mathrm{C}_{15} \mathrm{H}_{15} \mathrm{FN}_{4} \mathrm{O}_{4}$ : C, 53.89; $\mathrm{H}, 4.52 ; \mathrm{N}, 16.76$; Found: $\mathrm{C}, 54.21 ; \mathrm{H}, 4.81$; N, 16.98 .

3-(Isoxazol-5-yl)-5-(2-chloro-4,6-dimethoxyphenyl)-4,5-dihydro-1H-pyrazole-1-carboxamide (4o): Yield 63\%; Molecular Weight 350.76; m.p. 277-279 ${ }^{\circ} \mathrm{C}$; IR $\left(\mathrm{KBr}, \mathrm{cm}^{-1}\right)$ : $1637(\mathrm{C}=\mathrm{N}), 1675(\mathrm{C}=\mathrm{O}), 3390\left(-\mathrm{NH}_{2}\right)$; $^{1} \mathbf{H}$ NMR $\left(400 \mathrm{MHz}, \mathrm{CDCl}_{3}, \mathrm{ppm}\right): \delta 3.02\left(1 \mathrm{H}, \mathrm{H}_{\mathrm{A}}, \mathrm{dd}, \mathrm{J}_{\mathrm{AX}}=3.6 \mathrm{~Hz}, \mathrm{dd}, \mathrm{J}_{\mathrm{AB}}=16 \mathrm{~Hz}\right), 3.79\left(1 \mathrm{H}, \mathrm{H}_{\mathrm{B}}, \mathrm{dd}, \mathrm{J}_{\mathrm{AB}}=16 \mathrm{~Hz}, \mathrm{dd}, \mathrm{J}_{\mathrm{BX}}=\right.$ $12 \mathrm{~Hz}), 5.34\left(1 \mathrm{H}, \mathrm{H}_{\mathrm{x}}, \mathrm{dd}, \mathrm{J}_{\mathrm{Ax}}=3.6 \mathrm{~Hz}, \mathrm{dd}, \mathrm{J}_{\mathrm{BX}}=12 \mathrm{~Hz}\right), 10.49\left(2 \mathrm{H}, \mathrm{s}, \mathrm{NH}_{2}, \mathrm{D}_{2} \mathrm{O}\right.$ exchangeable $), 3.45(3 \mathrm{H}, \mathrm{s}, \mathrm{Ar}-$ $\left.\mathrm{OCH}_{3}\right), 3.95$ (3H, s, 2x Ar-OCH 3$), 6.45-8.20(4 \mathrm{H}, \mathrm{Ar}-\mathrm{H}) ;{ }^{13} \mathrm{C} \mathrm{NMR}$ (100 MHz, CDCl3, ppm): $\delta 155.92$ (C-3), 38.91 (C-4), 57.20 (C-4), $155.26\left(\mathrm{CONH}_{2}\right), 150.11$ (C-3 of Isoxazolyl ring), 100.21 (C-4 of Isoxazolyl ring), 158.76 (C-5 of Isoxazolyl ring), 120.76 (C-1 of phenyl ring), 134.25 (C-2 of phenyl ring), 107.22 (C-3 of phenyl ring), 161.08 (C-4 of phenyl ring), 98.35 (C-5 of phenyl ring), 158.28 (C-6 of phenyl ring), 55.8 (carbon of $-\mathrm{OCH}_{3}$ ), 56.12 (carbon of $\left.-\mathrm{OCH}_{3}\right)$. MS $(\mathrm{m} / \mathrm{z}, \%)$ : $335.1(\mathrm{M}+1,99.03)$; MS $(\mathrm{m} / \mathrm{z}, \%)$ : $351.08(\mathrm{M}+1,99.15)$; Anal. Calcd for: $\mathrm{C}_{15} \mathrm{H}_{15} \mathrm{ClN}_{4} \mathrm{O}_{4}$ : C, 51.36; H, 4.31; N, 15.97; Found: C, 52.11; H, 4.65; N, 16.11.

\subsection{In vitro antitubercular activity}

The preliminary antitubercular activity for the test compounds (4a-4o) was obtained against $M$. tuberculosis $\mathrm{H}_{37} \mathrm{Rv}$ strain. The MIC of all the compounds was determined by broth dilution assay [28-29] and 
is defined as the lowest concentration of the drug, which inhibits $\leq 99 \%$ of bacterial population present at the commencement of the assay. A frozen culture in Middlebrook 7H9 broth supplemented with 10\% albumindextrose-catalase and $0.2 \%$ glycerol was thawed and diluted in broth to $10^{5} \mathrm{cfu} \mathrm{mL}^{-1}$ (colony forming unit/mL) dilutions. The test compounds were dissolved in DMSO and then diluted in broth twice at the desired concentration. The final concentration of DMSO in the assay medium was $1.3 \%$. Each U-tube was then inoculated with $0.05 \mathrm{~mL}$ of standardized culture and then incubated at $37^{\circ} \mathrm{C}$ for 21 days. The growth in the Utubes was compared with visibility against positive control (without drug), negative control (without drug and inoculum) and with the standard isoniazid.

\subsection{Cytotoxicity studies}

The most potent compounds $\mathbf{4 n}$ and $\mathbf{4 o}$ of the series were tested in vitro for their cytotoxic properties against L02 (human normal cell line) by using MTT assay Mosmann's method. The MTT assay is based on the reduction of the soluble MTT $\left(0.5 \mathrm{mg} \mathrm{mL}^{-1}, 100 \mu \mathrm{L}\right)$, into a blue-purple formazan product, mainly by mitochondrial reductase activity inside living cells (Mosmann $\mathrm{T}$ et al., 1983). The cells used in cytotoxicity assay were cultured in RPMI 1640 medium supplemented with $10 \%$ fetal calf serum, penicillin, and streptomycin at $37{ }^{\circ} \mathrm{C}$ and humidified at $5 \% \mathrm{CO}_{2}$. Briefly cells were placed on 96-well plates at $100 \mu \mathrm{L}$ total volume with density of $1-2.5 \times 10^{4}$ cells per $\mathrm{mL}$ and were allowed to adhere for $24 \mathrm{~h}$ before treatment with tested drugs in DMSO solution $\left(10^{-5}, 10^{-6}, 10^{-7} \mathrm{~mol} \mathrm{~L}^{-1}\right.$ final concentration). Triplicate wells were treated with media and agents. Cell viability was assayed after $96 \mathrm{~h}$ of continuous drug exposure with a tetrazolium compound. The supernant medium was removed, and $150 \mu \mathrm{L}$ of DMSO solution was added to each well. The plates were gently agitated using mechanical plate mixer until the colour reaction was uniform and the OD570 was determined using micro plate reader. The $50 \%$ inhibitory concentration $\left(\mathrm{IC}_{50}\right)$ was defined as the concentration that reduced the absorbance of the untreated wells by $50 \%$ of vehicle in the MTT assay. Assays were performed in triplicate on three independent experiments. The results had good reproducibility between replicate wells with standard errors below $10 \%$.

Author contributions: Concept - P.K., V.R.K., A.B.S.; Design - P.K., V.R.K., A.B.S.; Supervision - V.R.K..; Resource P.K.; Materials - P.K.,; Data Collection and/or Processing - P.K., A.B.S.; Analysis and/or Interpretation - P.K., V.R.K., A.B.S.; Literature Search - P.K., A.B.S.; Writing - P.K., V.R.K., A.B.S.; Critical Reviews - P.K., V.R.K., A.B.S.

Conflict of interest statement: The authors declared no conflict of interest in the manuscript.

\section{REFERENCES}

[1] WHO Global tuberculosis report 2017. http://www.who.int/mediacentre/news/releases/2017/politicalcommitment-tb/en/ (accessed on November 02, 2017).

[2] Varghese B, Al-Busafi SN, Suliman FO, Al-Kindy SMZ. Unveiling a versatile heterocycle: pyrazoline-a review. RSC Adv. 2017; 7(74): 46999-47016. [CrossRef]

[3] Bhutani R, Pathak DP, Husain A, Kapoor G, Kant R. A review on recent development of pyrazoline as a pharmacologically active molecule. Int J Pharm Sci Res. 2015; 6(10): 4113-4128. [CrossRef]

[4] Sharma S, Sandeep K, Tania B, Jyoti G. Review on synthesis of bioactive pyrazoline derivatives. Chem Sci Trans. 2014; 3(3): 861-875. [CrossRef]

[5] Akranth M, Mohammed RA, Mohammed TA, Rikta S, Omprakash T, Mymoona A, Mohammed S, Mumtaz MA. Pyrazolines: A Biological Review. Mini Rev Med Chem. 2013; 6(13): 921-931. [CrossRef]

[6] Suresh K, Sandhya B, Sushma D, Rajiv K, Himanshu G. Biological activities of pyrazoline derivatives-A recent development. Recent Pat Antiinfect Drug Discov. 2009; 4(3). 154-163. [CrossRef]

[7] Mishra VK, Mishra M, Kashaw V, Kashaw SK. Synthesis of 1,3,5-trisubstituted pyrazolines as potential antimalarial and antimicrobial agents. Bioorg Med Chem. 2017; 25(6): 1949-1962. [CrossRef]

[8] Viveka S, Dinesha, Shama P, Nagaraja GK, Ballav S, Kerkar S. Design and synthesis of some new pyrazolylpyrazolines as potential anti-inflammatory, analgesic and antibacterial agents. Eur J Med Chem. 2015; 101: 442-451. [CrossRef]

[9] Rostom SA, Badr MH, Abd El Razik HA, Ashour HM, Abdel Wahab AE.Synthesis of some pyrazolines and pyrimidines derived from polymethoxy chalcones as anticancer and antimicrobial agents. Arch Pharm (Weinheim). 2011; 344(9): 572-587. [CrossRef] 
[10] Küçükoğlu K, Oral F, Aydın T, Yamalı C, Algül Ö, Sakagami H, Gülçin İ, Supuran CT, Gül Hİ. Synthesis, cytotoxicity and carbonic anhydrase inhibitory activities of new pyrazolines. J Enzym Inhib Med Chem. 2016; 31(sup4): 20-24. [CrossRef]

[11] George RF, Fouad MA, Gomaa IEO. Synthesis and cytotoxic activities of some pyrazoline derivatives bearing phenyl pyridazine core as new apoptosis inducers. Eur J Med Chem. 2016; 112: 48-59. [CrossRef]

[12] Montoya A, Quiroga J, Abonia R, Nogueras M, Cobo J, Insuasty B. Synthesis and in vitro antitumor activity of a novel series of 2-pyrazoline derivatives bearing the 4-aryloxy-7-chloroquinoline fragment. Molecules. 2014; 19(11): 1865618675. [CrossRef]

[13] Muneera MS, Joseph J. Design, synthesis, structural elucidation, pharmacological evaluation of metal complexes with pyrazoline derivatives. J Photoch Photobio B. 2016; 163: 57-68. [CrossRef]

[14] Abid M, Azam A. 1-N-substituted thiocarbamoyl-3-phenyl-2-pyrazolines: synthesis and in vitro antiamoebic activities. Eur J Med Chem. 2005; 40(9): 935-942. [CrossRef]

[15] Bandgar BP, Adsul LK, Chavan HV, Jalde SS, Shringare SN, Shaikh R, Meshram RJ, Gacche RN, Masand V. Synthesis, biological evaluation, and docking studies of 3-(substituted)-aryl-5-(9-methyl-3-carbazole)-1H-2-pyrazolines as potent anti-inflammatory and antioxidant agents. Bioorg Med Chem Lett. 2012; 22(18): 5839-44. [CrossRef]

[16] Bhosle MR, Deshmukh AR, Pal S, Srivastava AK, Mane RA. Synthesis of new thiazolylmethoxyphenyl pyrimidines and antihyperglycemic evaluation of the pyrimidines, analogues isoxazolines and pyrazolines. Bioorg Med Chem Lett. 2015; 25(11): 2442-2446. [CrossRef]

[17] Acharya BN, Saraswat D, Tiwari M, Shrivastava AK, Ghorpade R, Bapna S, Kaushik MP. Synthesis and antimalarial evaluation of 1,3, 5-trisubstituted pyrazolines. Eur J Med Chem. 2010; 45(2): 430-438. [CrossRef]

[18] Gul HI, Mete E, Taslimi P, Gulcin I, Supuran CT. Synthesis, carbonic anhydrase I and II inhibition studies of the 1,3,5trisubstituted-pyrazolines. J Enzyme Inhib Med Chem. 2017; 32(1): 189-192. [CrossRef]

[19] Dixit SR, Joshi SD, Kulkarni VH. Pyrrolyl pyrazoline carbaldehydes as enoyl-ACP reductase inhibitors: Design, synthesis and antitubercular activity. The Open Med Chem J. 2017; 11: 92-108. [CrossRef]

[20] Sharad KC, Vishal BP, Parth T, Vasudev T, Raval DK. Novel morpholinoquinoline nucleus clubbed with pyrazoline scaffolds: Synthesis, antibacterial, antitubercular and antimalarial activities. Eur J Med Chem. 2016; 112: $270-279$. [CrossRef]

[21] Ahsan MJ, Saini V. Design and synthesis of 3-(4-aminophenyl)-5-(4-methoxyphenyl)-4,5-dihydro-1H-pyrazole-1carboxamide/carbothioamide analogues as antitubercular agents. Beni-Suef J Bas App Sci. 2015; 4(1): 41-46. [CrossRef]

[22] Aftab A, Asif H, Shah AK, Mohammed M, Anil B. Synthesis, antimicrobial and antitubercular activities of some novel pyrazoline derivatives. J Saudi Chem Soc. 2016; 20(5): 577-584. [CrossRef]

[23] Gupta RA, Kashedikar SG. Synthesis, antitubercular activity, and QSAR analysis of substituted nitroaryl analogs: Chalcone, pyrazole, isoxazole, and pyrimidines. Med Chem Res. 2013; 22(8): 3863-3880. [CrossRef]

[24] Ahsan MJ, Samy JG, Khalilullah H, Bakht MA, Hassan MZ.Synthesis and antimycobacterial evaluation of 3a,4dihydro-3H-indeno [1,2-c] pyrazole-2-carboxamide analogues. Eur J Med Chem. 2011; 46(11): 5694-5697 . [CrossRef]

[25] Pallepati K, Venkata RK, Afzal BS. Antitubercular evaluation of isoxazolyl chalcones. Res J Pharm Biol Chem Sci. 2017; 8(6): 730-735.

[26] Rajendra Prasad Y, Lakshmana Rao A, Prasoona L, Murali K, Ravi Kumar P. Synthesis and antidepressant activity of some 1,3,5-triphenyl-2-pyrazolines and 3-(2"-hydroxynaphthalen-1"-yl)-1,5-diphenyl-2-pyrazolines. Bioorg Med Chem Lett. 2005; 15(22): 5030-5034. [CrossRef]

[27] Khode S, Maddi V, Aragade P, Palkar M, Ronad PK, Mamledesai S, Thippeswamy AH, Satyanarayana D. Synthesis and pharmacological evaluation of a novel series of 5-(substituted)aryl-3-(3-coumarinyl)-1-phenyl-2-pyrazolines as novel anti-inflammatory and analgesic agents. Eur J Med Chem. 2009; 44(4): 1682-1688. [CrossRef]

[28] Hearn MJ. PCT Int. Appl. 2002, WO 02043668; Chem. Abstr. 2002; 137:20296.

[29] Goto S, Jo K, Kawakita T, Misuhashi S, Nishino T, Ohasawa N, Tanami N. Determination method of minimum inhibitory concentrations. Chemotherapy 1981; 29: 76-79. 